\title{
Electric Parameter Extractions Using a Broadband Technique from Coaxial Line Discontinuities
}

\author{
F. Moukanda Mbango" ${ }^{1}$ F. Ndagijimana \\ ${ }^{1}$ Marien Ngouabi University, School of Sciences and Techniques, \\ BP: 69, Brazzaville, Congo Republic \\ ${ }^{2}$ Departement of Electrical Engineering, Univ. Grenoble Alpes, CNRS, Grenoble INP, G2Elab, \\ 151, Rue de la Papeterie, Saint-Martin d'Hères, F-38400, France
}

\begin{abstract}
:
We have proposed a simple technique for extracting high frequency material complex relative permittivity from $0.1 \mathrm{GHz}$ up to beyond $5.1 \mathrm{GHz}$ despite the test fixture's discontinuities along with the characteristic impedance. The frequency range limitation depends on the test fixture's sizes. Based on the S-parameter measurements, the overall technique associates the test fixture in which the sample to characterize is inserted and the technique for altering the propagation constant of the considered region. Mathematics concepts, through their formulations, allow extracting material complex relative permittivity. The technique foundation is primarily based on the fixture calibration when there is a filling up of vacuum. A coaxial test cell is used to validate the extraction procedure. That fixture is suitable for measuring material electric parameters used in medical and civil engineering, telecommunications, and oil and gas domains. We have presented results ensuing from measures of spring water and biological materials: human tissue and liquid $900 \mathrm{MHz}$. Both biological materials are liquids and react as a muscle subjected to an electromagnetic wave. The technique is broadband, making it easy to fill up the test cell with the sample to be tested.
\end{abstract}

Keywords: Complex permittivity, discontinuity, liquid measurement, transmission-line.

\section{Introduction}

Electromagnetic material characterization is the radiofrequency and microwave electronics element that relates to the analysis and interpretation of material behavior following electromagnetic excitation. All methods, along with their techniques, are based on two main principles: the entrapment of a material under test (MUT) in an environment called testing cell and the S-parameter measurements [1]-[7]. One of those techniques is the transmission line, which can be used in reflection and/or transmission [8]. In this paper, we have developed that technique (despite the fixture discontinuities) [9] to extract the complex relative permittivity $\varepsilon_{r}^{*}$ of some liquid materials in the frequency range $0.1-5.1 \mathrm{GHz}$ with accuracy better than 5\%. Many scientists have been developing techniques to sort out the discontinuity problems when computing data of intrinsic material parameters that generate order modes spreading inside of the test cell [10]-[11]. The developed technique in this paper is focused on the determination of the propagation constant $\gamma$, which is the best way to reduce the discontinuities' impacts [12]-[14]. The technique description is divided into two main parts: the mathematical formulation and the experimental validation with a second principle to counter difficulties of extracting sample losses. Experimental measurements, using some biological materials (900MHz liquid and human tissue) and normal water, have been done to validate the technique's procedure. We used a brass circular coaxial transmission line, in which the sample under test (SUT) is trapped. Also, O-rings have been set up in the fixture's design in order to avoid liquid spreading out.

\section{Theory and Mathematic Model \\ 2.1 Propagation constant}

The extraction of intrinsic electric parameters is quite often based on the use of the propagation 
constant $\gamma$ [11]-[14] when assuming that the MUT is none magnetic. This is due to discontinuities related to the transition interfaces, which are located between the input source and the ideal transmission line. This transmission-line secondary parameter is measured from S-parameters via the wave cascade matrix (WCM), written as [C] and given by:

$[C]=\frac{1}{S_{21}}\left[\begin{array}{cc}1 & -S_{22} \\ S_{11} & -\operatorname{det}(S)\end{array}\right]$

However, this matrix can be defined according to the transmission line propagation constant $\gamma$ on the assumption that $S_{11}=0$ and $S_{22}=0$ as:

$\left[C_{l_{1}}\right]=\left[\begin{array}{cc}e^{\gamma_{1} l_{1}} & 0 \\ 0 & e^{-\gamma_{1} l_{1}}\end{array}\right]=\left[\begin{array}{cc}\lambda & 0 \\ 0 & \frac{1}{\lambda}\end{array}\right]$

where $l$ is the line's length. Consider the fixture's schema as showed in figure 1, the discontinuity inverse transfer matrix is:

$\left[C_{\Gamma}\right]=\frac{1}{(1-\Gamma)}\left[\begin{array}{cc}1 & \Gamma \\ \Gamma & 1\end{array}\right]$

where

$\Gamma=\frac{Z_{c}-Z_{0}}{Z_{c}+Z_{0}}$

The total wave amplitude matrix for the device in figure 1 is written as:

$\left[C_{T o t}\right]=\left[\begin{array}{cc}e^{\gamma_{0} l_{0}} & 0 \\ 0 & e^{-\gamma_{0} l_{0}}\end{array}\right]\left[C_{\Gamma}\right]\left[\begin{array}{cc}e^{\gamma_{1} l_{1}} & 0 \\ 0 & e^{-\gamma_{1} l_{1}}\end{array}\right]\left[C_{\Gamma}\right]^{-1}\left[\begin{array}{cc}e^{\gamma_{0} l_{0}} & 0 \\ 0 & e^{-\gamma_{0} l_{0}}\end{array}\right]$

which gives

$\left[C_{T o t}\right]=\frac{1}{\left(1-\Gamma^{2}\right) e^{\gamma_{1} l_{1}}}\left[\begin{array}{cc}\left(e^{2 \gamma_{1} l_{1}}-\Gamma^{2}\right) e^{2 \gamma_{0} l_{0}} & \Gamma\left(1-e^{2 \gamma_{1} l_{1}}\right) \\ -\Gamma\left(1-e^{2 \gamma_{1} l_{1}}\right) & \left(1-\Gamma^{2} e^{2 \gamma_{1} l_{1}}\right) e^{-2 \gamma_{0} l_{0}}\end{array}\right]$

Once the de-embedding is done, equation (5) is reduced to:

$\left[C_{R}\right]=\frac{1}{\left(1-\Gamma^{2}\right) e^{\gamma_{1} l_{1}}}\left[\begin{array}{cc}\left(e^{2 \gamma_{1} l_{1}}-\Gamma^{2}\right) & \Gamma\left(1-e^{2 \gamma_{1} l_{1}}\right) \\ -\Gamma\left(1-e^{2 \gamma_{1} l_{1}}\right) & \left(1-\Gamma^{2} e^{2 \gamma_{1} l_{1}}\right)\end{array}\right]$
From equation (4), the characteristic impedance of the cell test is given as:

$Z_{c}=Z_{0}\left(\frac{1+\Gamma}{1-\Gamma}\right)$

Figure 1 is the summary of the entire testing cell when the sample under test (SUT) is inside or not.

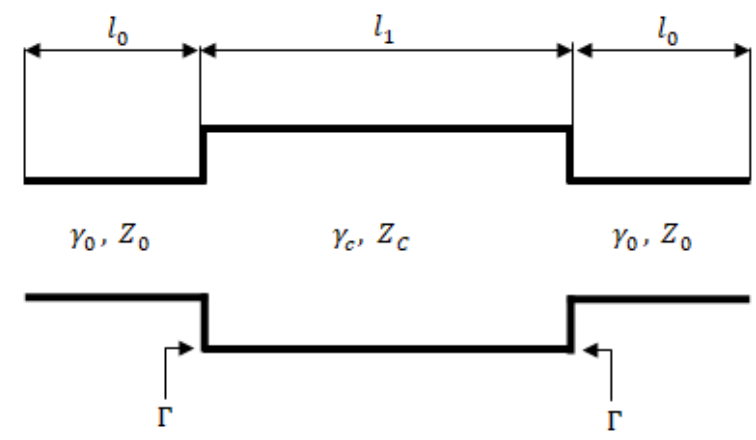

Figure 1: Simplified representation of a transmissionline in the presence of discontinuities.

The wave cascade matrix of the ideal line is given by the following equation:

$\left[C_{l_{1}}\right]=\left[C_{\Gamma}\right]^{-1}\left[C_{R} \llbracket C_{\Gamma}\right]$

Solving equation (8) leads to:

$\left[C_{l_{1}}\right]=\frac{1}{1-\Gamma^{2}}\left[\begin{array}{cc}\left\{C_{11}+\Gamma C_{12}-\Gamma\left(C_{21}+\Gamma C_{22}\right)\right\} & B \\ \Gamma C_{22}+C_{21}-\Gamma\left(C_{11}+\Gamma C_{12}\right) & D\end{array}\right]$

$$
\begin{aligned}
& B=\left\{\Gamma C_{11}+C_{12}-\Gamma\left(C_{22}+\Gamma C_{21}\right)\right\} \\
& D=\left\{C_{22}+\Gamma C_{21}-\Gamma\left(C_{12}+\Gamma C_{11}\right)\right\}
\end{aligned}
$$

Using equation (2), by identification, we need to solve the second-order polynomial, which is:

$$
C_{21} \Gamma^{2}+\left(C_{22}-C_{11}\right) \Gamma-C_{12}=0
$$

We get easily the reflection coefficient at the lineconnector interface. It is given as:

$\Gamma_{1,2}=\frac{\left(C_{11}-C_{22}\right) \mp \sqrt{\left(C_{11}-C_{22}\right)^{2}+4 C_{12} C_{21}}}{2 C_{21}}$ 
This last result is used for determining the characteristic impedance expressed in equation (4). From equation (10), combined with (2), we obtain the propagation constant expression as:

$\gamma_{1} l_{1}=\ln \left\{\frac{C_{11}+\Gamma C_{12}-\Gamma\left(C_{21}+\Gamma C_{22}\right)}{1-\Gamma^{2}}\right\}$

As far as we have considered the fixture as the ideal one, it is necessary that $\Gamma_{v, m}=0$. In that case, the propagation constant in two situations (vacuum and SUT) becomes:

$\left(\gamma_{1} l_{1}\right)_{v, m}=\ln \left\{\left(C_{11}\right)_{v, m}\right\}$

We ultimately rewrote the propagation constant in both situations: in or out of presence of the sample under test, indicated by $v$ for vacuum and $m$ for material under test. The phase constant in the case of vacuum is determined by $(\beta l)_{v}=\operatorname{Im}\left(\ln \left\{\left(C_{11}\right)_{v}\right\}\right)$ while $(\beta l)_{m}=\operatorname{Im}\left(\ln \left\{\left(C_{11}\right)_{m}\right\}\right)$. This constant is obtained after having disrupted the environment of the testing cell. Moreover, the energy conservation equation is often used in telecommunication as:

$R+T=1$

It allows settling up loss problems by considering a general mismatched system and easily demonstrating that:

$\left(\alpha l_{(N p)}\right)_{v, m}=\frac{\left(-\left(S_{21}^{d B}\right)_{v, m}+\left(S_{11}^{d B}\right)_{v, m}+10 \log \{H\}\right)}{20 \log (e)}$

where

$H=\frac{1}{\left|\left(S_{11}\right)_{v, m}\right|^{2}}-1$

In the particular case where the system is matched, equation (14) becomes:

$\left(\alpha l_{(N p)}\right)_{v, m}=-\left(S_{21}^{d B}\right)_{v, m}$

$S_{11}$ and $S_{21}$ are found from equation (2), as results are given below:
$\left\{\begin{array}{l}\left(S_{11}\right)_{v, m}=\frac{\left(C_{21}^{\prime}\right)_{v, m}}{\left(C_{11}^{\prime}\right)_{v, m}} \\ \left(S_{21}\right)_{v, m}=\frac{1}{\left(C_{11}^{\prime}\right)_{v, m}}\end{array}\right.$

Making measurements of connections in short circuit and in open circuit, the obtained reflection parameters, respectively $S_{11}^{S C}$ and $S_{11}^{O C}$, give the opportunity for extracting the characteristic impedance and the propagation constant $\gamma_{0} l_{0}$ as:

$$
\begin{aligned}
& Z_{0}=Z_{n} \sqrt{\left(\frac{1+S_{11}^{S C}}{1-S_{11}^{S C}}\right) \cdot\left(\frac{1+S_{11}^{O C}}{1-S_{11}^{O C}}\right)} \\
& \gamma_{0} l_{0}=\tanh ^{-1} \sqrt{\left(\frac{1+S_{11}^{S C}}{1-S_{11}^{S C}}\right) \cdot\left(\frac{1-S_{11}^{O C}}{1+S_{11}^{O C}}\right)}
\end{aligned}
$$

where $Z_{n}=50 \Omega$ is the characteristic impedance of the metering equipment.

\subsection{Extraction of material electric parameters}

We have extracted the material intrinsic parameters: the relative permittivity $\varepsilon_{r}$, the dielectric loss tangent $\tan \delta_{d}$, and/or the electric conductivity $\sigma_{e}$. Mathematic expressions giving the methodology of these parameters have been implemented with a compute program. This is a real stake of several scientific works and publications [7]-[12]. After having linearised the phase constant, with the assumption that the SUT is a perfect insulator, equation (19) is expressed as:

$$
\left\{\begin{array}{c}
\varepsilon_{r}=\left[\frac{(\beta l)_{m}}{(\beta l)_{v}}\right]^{2} \\
\tan \delta_{d}=2\left[\frac{(\alpha l)_{m}}{(\beta l)_{m}}-\frac{(\alpha l)_{v}}{(\beta l)_{v}}\right]
\end{array}\right.
$$

and the electric conductor:

$$
\sigma_{e}=2 \omega \varepsilon_{0}\left[\frac{(\alpha l)_{m}}{(\beta l)_{m}} \varepsilon_{r}-\frac{(\alpha l)_{v}}{(\beta l)_{v}}\right]
$$




\section{Experiment Results and Discussion}

We calibrated the vector network analyzer in 2-port to solve all measurement system blemishes [15].

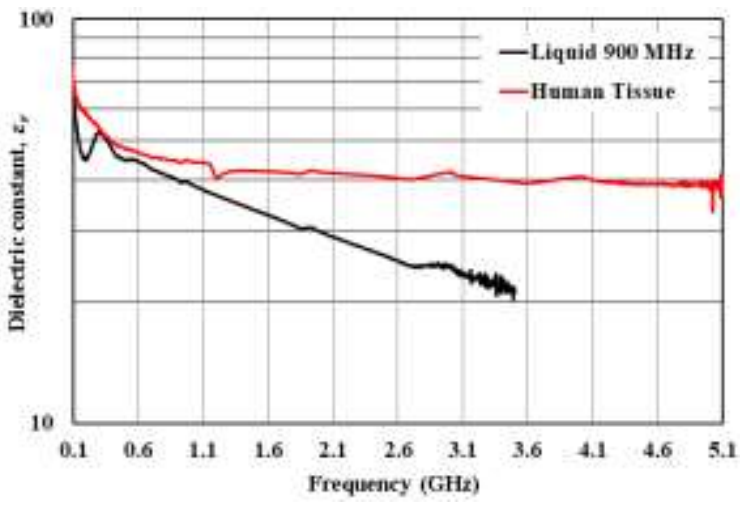

Figure 2a: Relative permittivity of human tissue and liquid $900 \mathrm{MHz}$

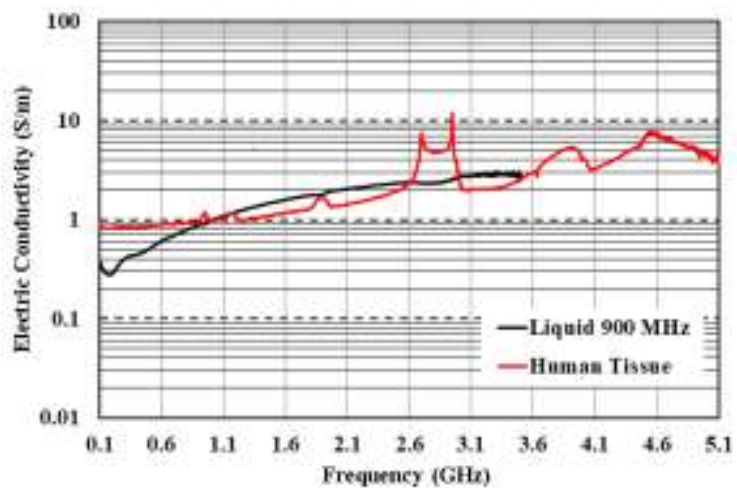

Figure 2b: Electric conductivity of human tissue and liquid $900 \mathrm{MHz}$

We compare the sound-transmission gel manufacturer values at $900 \mathrm{MHz}$ to those we obtained through the following table resume.

Table 1: Benchmarking values of liquid $900 \mathrm{MHz}$

\begin{tabular}{|c|c|c|}
\hline Parameters & Manufacturer & Measured \\
\hline$\varepsilon_{r}$ & 39.3 & 40.03 \\
\hline$\sigma_{e}(S / m)$ & 0.95 & 0.89 \\
\hline
\end{tabular}

We observed an error of $1.86 \%$ and $6.31 \%$ on the relative permittivity and the electric conductivity, respectively. We sketched curves to compare the human tissue electric parameters to validate the trend that has been observed with the soundtransmission gel.

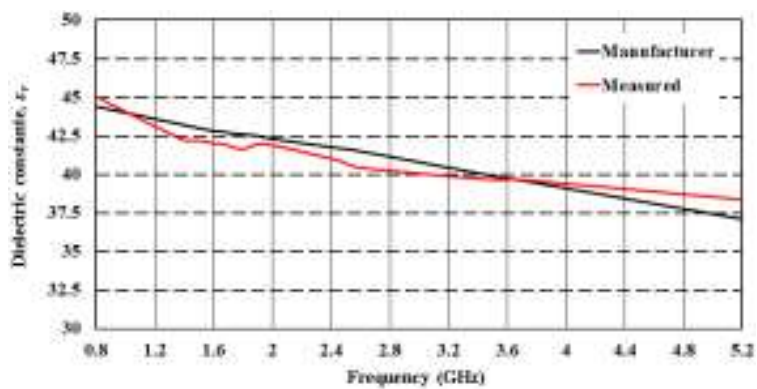

Figure 3a: Comparative results of human tissue relative permittivity

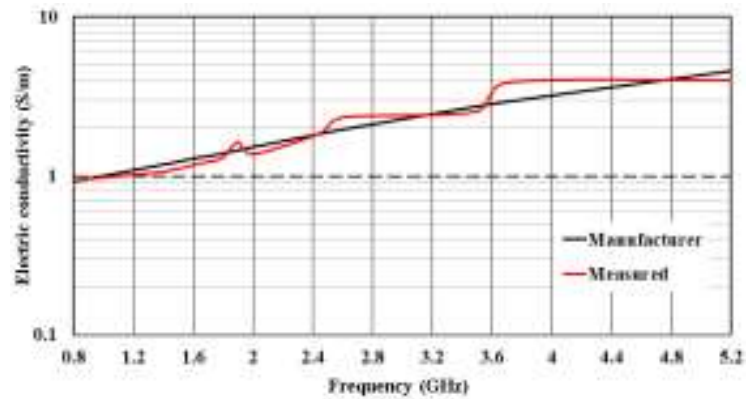

Figure 3b: Comparative results of human tissue electric conductivity of human tissue

These measured results through its sketches show a good match with the manufacturer ones.

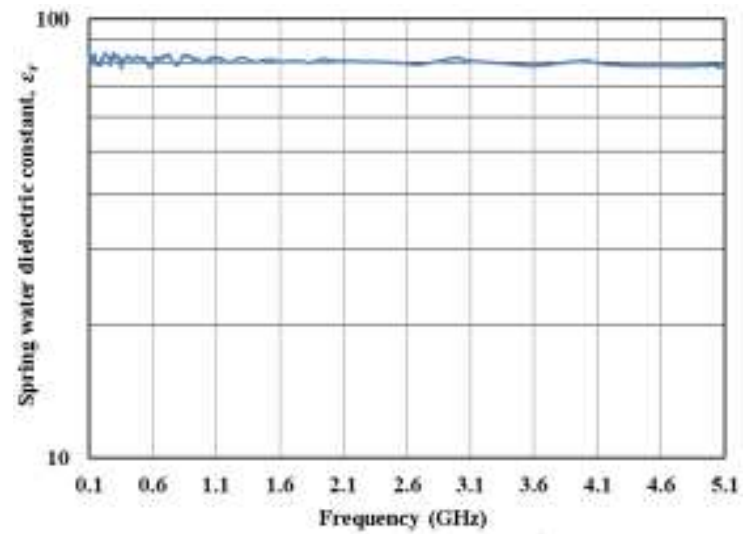

Figure 4a: Relative permittivity of spring water

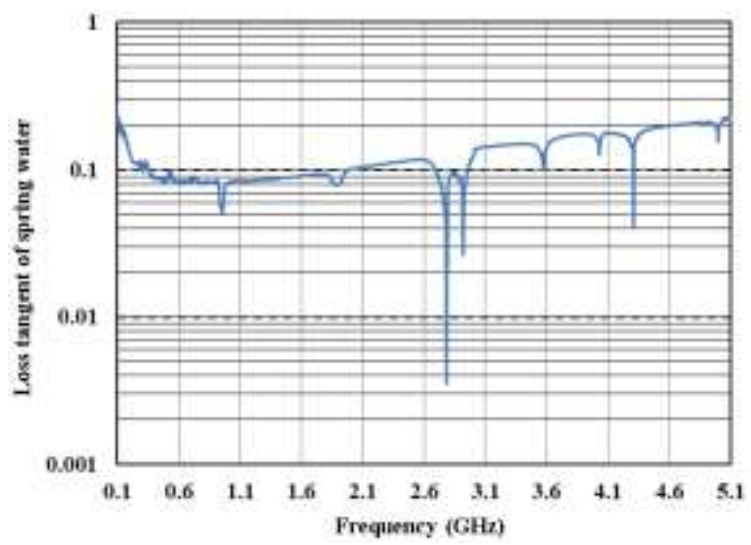

Figure 4b: Loss tangent of spring water 
The spring water results are in accordance with the two transmission-line technique [16].

\section{Conclusion}

A broadband technique for measuring any material bulk properties (i.e. complex permittivity) has been developed. The technique utilizes a coaxial transmission-line fixture, loaded with a material in the unknown intrinsic parameters. Those parameter measurements are made despite the fixture's discontinuities, and S-parameters are computed automatically with the network analyzer in order to be used in the extraction procedure. Some measured data for standard biological dielectrics in [0.1-5.2] $\mathrm{GHz}$ have been presented. The technique allows for accuracy better than $\pm 5 \%$ on relative permittivity and $\pm 10 \%$ on the electric conductivity parameters. The sample under test conductive losses and its dielectric constant along with the device dimensions are the three reasons that caused the frequency band limitations. From different obtained results, this measurement technique should be suitable for other materials with electromagnetic applications.

\section{References}

[1] A. Lewandowski et al., "0.05-3 GHz VNA Characterization of Soil Dielectric Properties Based on the Multiline TRL Calibration," Measurement Science and Technology. vol. 28, No. 2, pp. 1-7, 2017.

[2] M. Moukanda, F. Ndagijimana, J. Chilo, P. Saguet, "A Coaxial Probe Fixture Used for Extracting Complex Permittivity of Thin Layer," In Proceedings of the IEEE International Symposium on Signal, Systems and Electronics (ISSSE), pp. 431-434, 2007.

[3] Muhammed Said Boybay, Omar M. Ramahi, "Material Characterization Using Complementary Split-Ring Resonators," IEEE Transactions on Instrumentation and Measurement, vol. 61, No. 11, pp. 3039-3046, 2012.

[4] M. Aziz, A. K. Ermeey, K. Jusoff, "Nondestructive and Noncontact Dielectric Measurement Methods for Transformer Oil Using Free-space Microwave Measurement System in 19-25 GHz Frequency Range," International Journal on Computer Science and Engineering, vol. 02, No. 4, pp. 1184-1189, 2010.
[5] L. G. Da Silva, and al., "Electromagnetic Characterization of Aircraft Composite Materials and its Effects on the Antenna Performance," IEEE Journal of Microwaves, Optoelectronics and Electromagnetic Applications. vol. 16, No. 1, pp. 218-236, 2017.

[6] Olumide Ogunlade, Roger D. Pollard, Ian C. Hunter, "A New Method of Obtaining the Permittivity of Liquids Using In-Waveguide Technique," IEEE Microwave and Wireless Components Letters. vol. 16, No. 6, pp. 363365, 2006.

[7] M. Moukanda, F. Ndagijimana, J. Chilo, P. Saguet, "Complex Permittivity Extraction Using Two Transmission-line S-Parameter Measurements," The African Physical Review. vol. 2, No. 30, pp. 62-64, 2008.

[8] J. A. Reynoso-Hernandez and al., "A Method for Computing the Characteristic Impedance of Transmission Lines Using the Wave Cascade Matrix Formalism," In the proceedings of the IEEE 61st Automatic RF Techniques Group Conference Digest (ARFTG-CD), pp. 179-185, 2003.

[9] Ranajit Dey, S. B Chakrabarty, Rajeev Jyoti, Thomas Kurian, "Higher Order Mode Analysis of Dual-Post Discontinuity in a Circular Waveguide," IETE Journal of Research, vol. 62, No. 1, pp. 55- 62, 2016.

[10] J. A. Reynoso-Hernandez, "Unified Method for Determining the Complex Propagation Constant of Reflecting and Nonreflecting Transmission lines," IEEE Microwave and Wireless Components Letters, vol.13, No. 8, pp. 351-353, August 2003.

[11] N. Belhadj-Tahar, O. Meyer, A. FourrierLamer,"Broad-Band Microwave Characterization of Bi-layered Materials Using a Coaxial Discontinuity with Applications for Thin Conductive Films for Microelectronics and Material in Air-Tight Cell," IEEE Transactions on Microwave Theory and Techniques, vol. 45, No. 2, pp. 260-267, 1997.

[12] Nobuo Nagai, Takashi Yahagi, "Transmission circuit Theory Based on Eigen-Values of Cascade Matrix," Journal of Signal Processing, vol. 20, No. 3, pp. 113-120, 2016.

[13] H. Elmajid, J. Terhzaz, H. Ammor, M. Chaïbi, M. Sanchez, "Determination of the Complex Permittivity of Each Layer for a Bi-layer Dielectric Material Using Transmission (ABCD) Matrix in Ku-Band Frequency," Journal of Engineering Science and Technology Review, vol. 9, No. 1, pp. 61-65, 2016. 
[14] Nawfal Jebbor, Seddik Bri, “A Microwave Method for Complex Permittivity Extraction of Thin Materials," Journal of microwaves, Optoelectronics and Electromagnetic Applications, vol. 11, No. 2, 2012.

[15] Carmen Bachiller, and al., "Efficient technique for the Cascade Connection of Multiple TwoPort Scattering Matrices," Transaction on Microwave Theory and Techniques, vol. 55, No. 9, pp. 1880-1889, 2007.

[16] F. Moukanda Mbango, A. Al Takach, F. Ndagijimana, "Complex Relative Permittivity Extraction Technique of Biotechnology Materials in Microwave Domains," International Journal of Electronics, Communication \& Instrumentation Engineering Research and Development, vol. 9, accepted date: 30/04/2019.

\section{Author Profile}

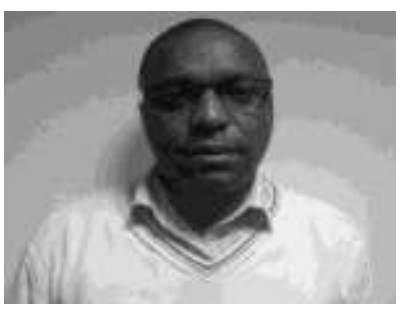

Franck Moukanda Mbango received the Engineer degree in microwave electronics from Mouloud Mammeri University, Algeria, in 2001. In 2003 and 2008, he respectively received the M.Sc. and the $\mathrm{PhD}$. degrees in microwave circuits from National Polytechnic Institute (INP) and Joseph FOURIER University at Grenoble, France. From 2009 to 2014, he was involved in several Telecommunication industrial projects as a R\&D Engineer and Research Assistant Consultant to ALTRAN Technologies for Vallourec, also as Electromagnetic Compatibility Engineer to CSTB. He currently works as CAMES Senior Lecturer and researcher at Université Marien Ngouabi, Congo. His fields of research are concerned with high-frequency material measurement techniques, microwave device EM modelling for wireless applications and electromagnetic environment impact, as well as sensor principles for radar systems and wireless solutions for communication systems.

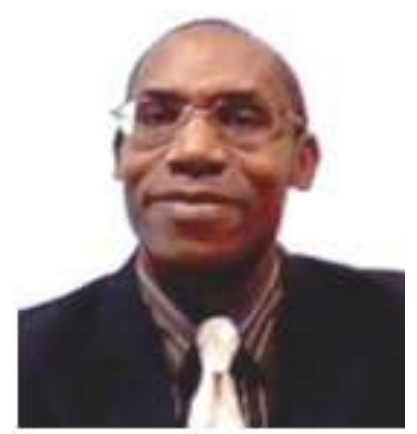

Fabien Ndagijimana received his $\mathrm{PhD}$, specializing in Microwave and optoelectronics, in December 1990, at Institut National Polytechnique de Grenoble (INPG) in France. He then joined the faculty of Electrical Engineering ENSERG as associate Professor where he teaches microwave techniques and electromagnetic modelling. Since September 1997, he joined the Universite Grenoble Alpes, were he is full professor at the Institut Universitaire de Technologie (IUT). He's now Professor at Université Grenoble Alpes in Grenoble-France. His research activity focuses on the characterization and electromagnetic modelling of microwave devices for wireless applications, signal integrity in high speed applications and test tools for electromagnetic compatibility standards. 